\title{
Low-Cost Measurement for a Secondary Mode S Radar Transmitter
}

\author{
Ángel Parra-Cerrada, Vicente González-Posadas, José Luis Jiménez-Martín, Álvaro Blanco-del-Campo, \\ Wilmar Hernandez, Senior Member, IEEE, and Carlos Calderón-Córdova
}

\begin{abstract}
A low-cost, multiple-purpose, and high-precision timing test setup for the measurements of secondary Mode S radar transmission signal was proposed. The goal was to fully guarantee compliance of the proposed transmitter under test with the really hard International Civil Aircraft Organization requirements using traditional measurement equipment, which was difficult or even impossible to ensure up to now. The low-cost structure proposed in this paper allows the user to perform measurements independently of the measurements performed by the pieces of test equipment shelled by the manufacturer of radar, which is a very important aspect since the independence of the verifications is a mandatory requirement established by the safety standards of civil aviation. The proposed setup has been used to verify several transmitters with some defects that are not detected by monopulse secondary surveillance radar specific pieces of test equipment that are focused on more high-level functionalities. It also is valid and it has been used, as a general-purpose setup, for testing other radio navigation aids.
\end{abstract}

Index Terms-Commercial-off-the-shelf (COTS) equipment, differential phase shift keying, envelope and phase measurements, global positioning system disciplined oscillator (GPSDO), Mode S radar, monopulse secondary surveillance radar (MSSR), radar measurements, transmitter measurements.

\section{INTRODUCTION}

$\mathbf{I}$ $\mathrm{N}$ THIS paper a low-cost and high-precision method to measure and analyze the most important parameters of the transmitter of a monopulse secondary surveillance radar (MSSR) is presented. In the scientific literature, there are several papers that analyze the performance of monopulse receivers [1], MSSR antennas [2], and spurious emissions of an

This work was supported in part by the Spanish Ministry of science and Eaucation under Project TEC2006-13248-C04-04/TCM, Project Consolider CSD2008-0068, and Project TEC2009-14525-C02-01, in part by the National Secretary of Higher Education, Science, Technology and Innovation of Ecuador through PROMETEO Project, and in part by the Technical University of Loja, Loja, Ecuador. The Associate Editor coordinating the review process was Dr. Matteo Pastorino.

Á. Parra-Cerrada, V. González-Posadas, and J. L. Jiménez-Martín are with the Department of Teoría de la Señal y Comunicaciones, Universidad Politécnica de Madrid, Madrid 28040, Spain (e-mail: angel.parra@upm.es; vicente.gonzalez@upm.es; joseluis.jimenez@upm.es).

Á. Blanco-del-Campo is with the Microwave Sensing, Signals and Systems Group, Department of Microelectronics, Delft University of Technology, Delft 2628CC, The Netherlands (e-mail: a.blancodelcamp@ tudelft.nl).

W. Hernandez and C. Calderón-Córdova are with the Department of Computer Science and Electronics, Universidad Técnica Particular de Loja, Loja 11432, Ecuador (e-mail: whernandez@utpl.edu.ec; cacalderon@utpl.edu.ec).
MSSR [3]. However, these papers do not deal with the up-link signal of the MSSR taking into consideration the modulation performances of this signal. This paper 34 shows a detailed analysis of the transmitted signal by an MSSR, allowing us to verify compliance with International Standards and Recommended Practices [4]. This verification is made by using a exible user-controlled method that is independent of any manufacturer of specific commercial test equipment, which in many cases is the same as the manufacturer of the radar. This method guarantees that problems cannot be undetected because of the same implementation of some hardware is used in transmitter and test equipment.

Nowadays, the number of MSSR is increasing very significantly and a further increase is expected in the coming years [5]. In addition, due to the fact that the MSSR is a critical point in aviation safety, it is of utmost importance to have diagnostic capabilities of the MSSR independently of the manufacturer of the radar [6] and as a complement to the built-in test equipment of the radar, having a low-cost system that uses light and easily transportable equipment that allows checking in situ in remote areas in an affordable manner.

During the fifties, the United States of America required all aircraft entering its airspace to be identified by means of secondary surveillance radar (SSR), compatible with the military identification friend or foe (IFF) system IFF Mark X selective identification feature system [7]. Due to its widespread use, it was established as a reliable radar sensor for air traffic control (ATC), although with some technical limitations, such as garble, fruit, track wander, and multipath propagation. For this reason, some improvements were made, for example, monopulse in azimuth (eliminating track wander) [8], greater vertical aperture antennas (reducing multipath cancelation and silence cone) [9], and sidelobe suppression system improvement. All these improvements allowed this ATC system to become the most world-wide used one [10]. Nevertheless, once these improvements were achieved, more improvements in SSR systems were required, such as selective modes of interrogation and getting a better data link between radar ground station and aircraft [11]. These improvements were achieved by modulating not only amplitude of the transmitted uplink signal but also its phase. This way, Mode $\mathrm{S}$ radar was on track.

Furthermore, as it was impossible to renew at once all the systems that were already in use worldwide, the new system had to be capable of responding and transmitting to older versions of IFF, and this is the reason why Mode $S$ radars transmitted signals and systems themselves became more and 
more complicated, fulfilling hard requirements in amplitude, phase, and time synchronization as well. It is a well-known fact that whatever system is used to control, manufacture, or develop anything in which human lives can be involved and it has to get through a very strict certification process. Therefore, amplitude, phase, frequency, and timing conditions of Mode $S$ radars must be measured and certified [12], involving specific and quite expensive equipment as well as high technical staff to measure them. The manufacturer is usually the responsible of the certification process, but each piece of equipment must be verified periodically by the user as it is required by each national ATC normative. This is normally requires once a year.

This paper emphasizes this key point and shows a novel method of Mode $\mathrm{S}$ radar ground transmitter measurements using only the low-cost commercial-off-the-shelf equipment. The proposed method provides a complete verification of the transmitter using a typical acquisition scheme. So, this verification is performed without any dependence with the radar manufacturer. In short, for timing requirements, a global positioning system (GPS) disciplined oscillator (GPSDO) is used [13], and for sampling and storing the signal for proper automatic processing, a high-frequency sampling oscilloscope is used [14]. In addition, a computer is used to get the data from the oscilloscope for analysis and signal processing. The processing of the signal on a computer using MATLAB allows the specific and particular parameter monitoring that each user desires independent of the interpretation that the manufacturer of the transmitter made (usually, it is the same interpretation that the manufacturer implements on the COST test equipment).

A prototype of the measurement system was implemented and it was used to carry out the measurements and the analysis of some signals of two defective transmitters.

The organization of this paper is as follows. The test setup is explained in Section II. The signal acquisition and processing is explained in Section III. Section IV is aimed at showing the experimental results. Finally, the main conclusions of this paper are summarized in Section V.

\section{TEST SETUP}

Usually, when trying to measure a waveform that is difficult to measure, the required and, therefore, used equipment is specifically designed for that purpose, and very often, the state of the art technology is involved in it. For this reason, equipment designed for this purpose is hard to find and expensive. However, sometimes designers can use inexpensive technologies to solve the problem of measuring waveforms with very complex characteristics [15], [16]. For example, not long ago, if real-time data acquisition and signal processing was involved, it was difficult and very expensive to save a large amount of data. But a wide range of digital oscilloscopes nowadays can do it [17].

To carry out Mode $\mathrm{S}$ radar transmitter measurements, two main conditions have to be met. First, a bandwidth of $70 \mathrm{MHz}(\mathrm{IF}+\mathrm{BW} / 2)$ has to be sampled and stored, although offline processing can be used. Second, a highly

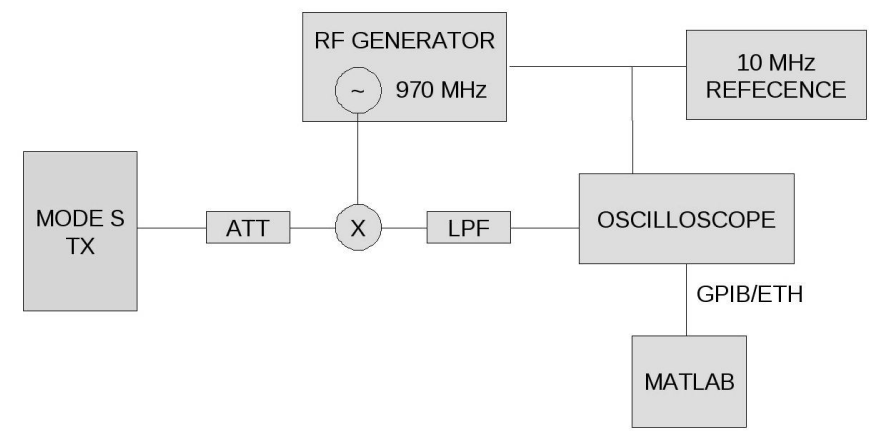

Fig. 1. Schematic of the proposed test setup.

stable reference signal has to be used for accurate phase and timing measurements.

To meet the first condition, this paper presents the test setup shown in Fig. 1, which uses the digital oscilloscope TDS3034C as a data acquisition system. Its sampling rate $(2.5 \mathrm{G} / \mathrm{s})$ is high enough to manage the required bandwidth and to store the needed information for a simple and unique interrogation.

To meet the second condition, a GPSDO is used. This is shown in Fig. 2 and its performance is explained in Section II-B.

The test setup shown in Fig. 1 uses an intermediate frequency (IF) of $60 \mathrm{MHz}$. This frequency is a typical IF for SSR systems and is low enough to be used in many mediumrange digital oscilloscopes. Being a typical frequency makes it easy to purchase filters, attenuators, mixers, and so on at low prices.

Using a digital oscilloscope along with a computer to control it and process the signal becomes a low-cost and high-performance signal analysis system, good enough for our purposes. Therefore, the proposed test setup for the Mode S radar transmission measurements consists of the following: 1) attenuator; 2) passive mixer; 3) low-pass filter; 4) $10-\mathrm{MHz}$ GPS oscillator reference; 5) signal generator; 6) digital oscilloscope; 7) desktop computer; and 8) MATLAB. At this point, it is important to say that direct conversion of the IF signal is being used because of its inherent advantages compared with the use of a traditional I/Q (or I/Q and video) demodulator used in most transponders. Some of the main advantages are that amplitude to I/Q time delay, amplitude mismatch between I/Q branches, and I/Q phase unbalance errors are avoided.

\section{A. Signal Parameters}

As it has been said before, when Mode $S$ radars are used, human lives are involved and security becomes a must. Thus, the requirements of the signal parameters of a Mode S interrogation are difficult to meet, to ensure proper operation and that the signal complies with the International Civil Aircraft Organization (ICAO) [4] requirements. These parameters do not only define spectrum and amplitude, but also intrapulse phase evolution, amplitude variation in 


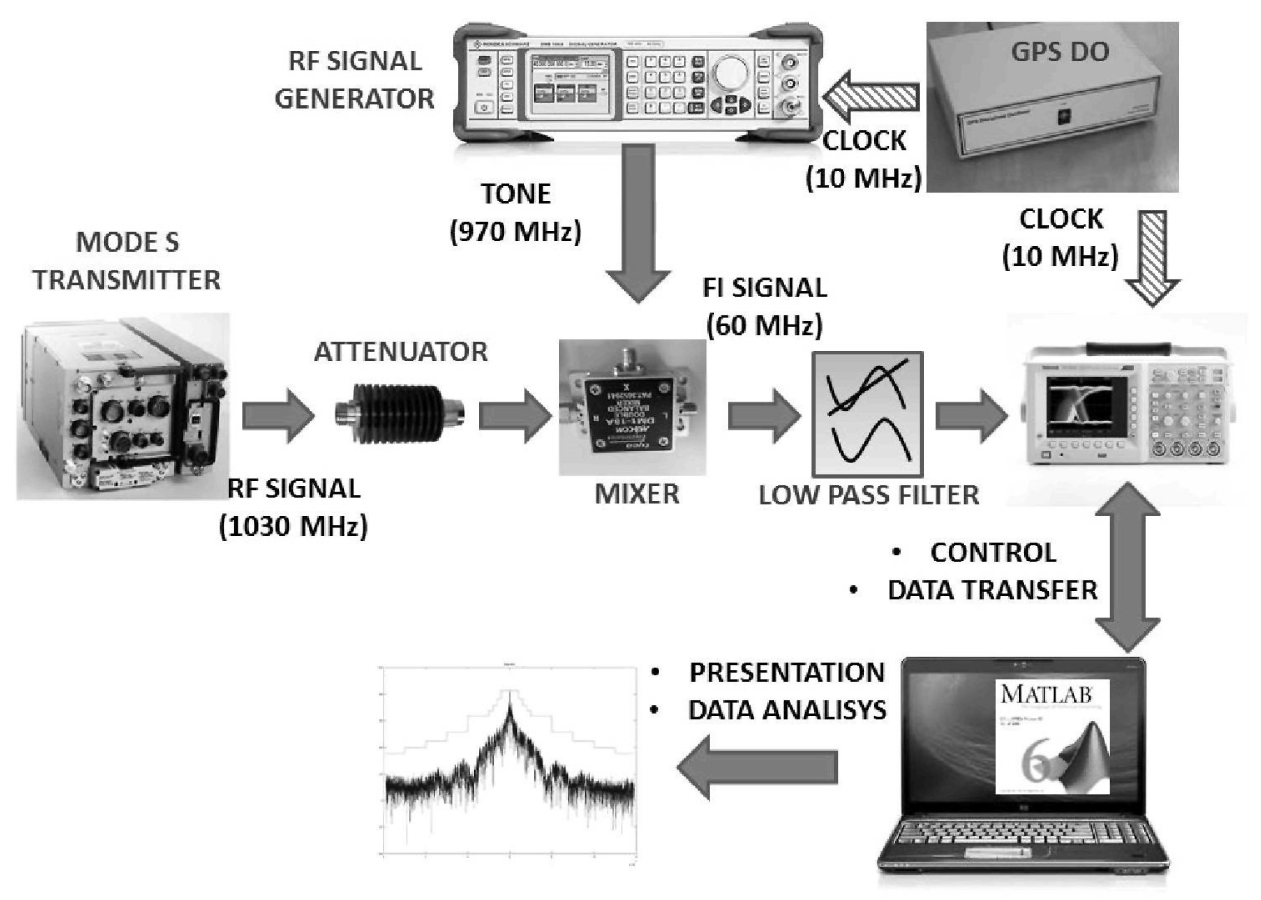

Fig. 2. Test setup.

each data bit, pulse duration, and so on. The parameters that define a Mode $\mathrm{S}$ interrogation are shown in Table I.

Most tight requirements are related to timing conditions. Timing parameters are defined for amplitude (e.g., rise time and width) and amplitude to signal phase, and also for phase evolutions. Measurements with a precision of nanoseconds are required because the tolerances are of the order of tens of nanoseconds, so timing setup becomes a very hard task to accomplish.

It is important to remark that all pieces of commercial offthe-shelf equipment known by the authors are mainly focused in the upper level information and operation of the MSSR. They are able to generate scenarios, targets with movement, garbling, and so on. But these pieces of test equipment use a demodulator to decode the interrogations and most of them are not able (really none of the equipment known by the authors) to detect problems in the modulation waveform. Therefore, they are able to decode the signal in a high $\mathrm{S} / \mathrm{N}$ condition, but they do not guarantee a proper decodification by the transponders of the aerial platforms in a realistic $S / N$ condition.

\section{B. Timing Setup}

In this paper, a GPSDO was used to obtain a highly stable reference signal. The principle of operation of a GPSDO is as follows. A GPSDO works by disciplining or steering a high-quality quartz or rubidium oscillator by locking the output to a GPS signal via a tracking loop mainly in two ways: either through a loop filter or via a microcontroller using software to compensate not only for phase and frequency of the signal but also for aging. Thus, stability in the long term and in the short term of the GPS signal is combined.
The short-term stability is given by the crystals of quartz or rubidium [18], [19], and it archives a frequency stability much better than the one required, $0.01 \mathrm{MHz}$ at $1030 \mathrm{MHz}$, for a 10-MHz reference signal.

The precision requirements defined by ICAO are in terms of frequency stability and position of data bits. The tolerance in frequency defined by ICAO is $\pm 0.01 \mathrm{MHz} @ 1030 \mathrm{MHz}$, and this is $9709 \mathrm{ppm}$. The requirement of position of the bits is $\pm 20 \mathrm{~ns}$ at a maximum distance of $28 \mu$ s with $714286 \mathrm{ppm}$. Therefore, the most restrictive factor is frequency stability. To measure the frequency with an uncertainty of $1 \%$ at the limit of the acceptable range defined by ICAO, it is required a stability of the system better than $97 \mathrm{ppm}$. The tolerance in timing is $\pm 20 \mathrm{~ns}$, so an oscilloscope with a sample rate of 2.5 GSampes/s provides a $2 \%$ accuracy. A higher sampling rate could be used in the case of requiring a more selective system for measuring transmitters near the specification limit.

The test setup does not require a phase-locked loop (PLL) to lock the frequency because signal requirements are referred to nominal transmitter frequency. Thus, a GPSDO is used to obtain an absolute 10-MHz reference. Most of the systems for aircrafts do not use a PLL to lock the signal, and instead, they use noncoherent receivers, because they must listen to multiple signal sources at the same time, replying to the highest priority one. Most systems use the arrival time as priority policy, and they reply to the first one and the others are thrown away. But in some more complex systems, those that include military functionalities, other policies are used to determine the reply priority and order.

As most of the signal parameters defined by ICAO [4] are related to the phase of the signal, the accuracy of measurement is improved using a GPSDO as reference signal $(10 \mathrm{MHz})$ for the generator $(970 \mathrm{MHz})$ and also for the oscilloscope that 


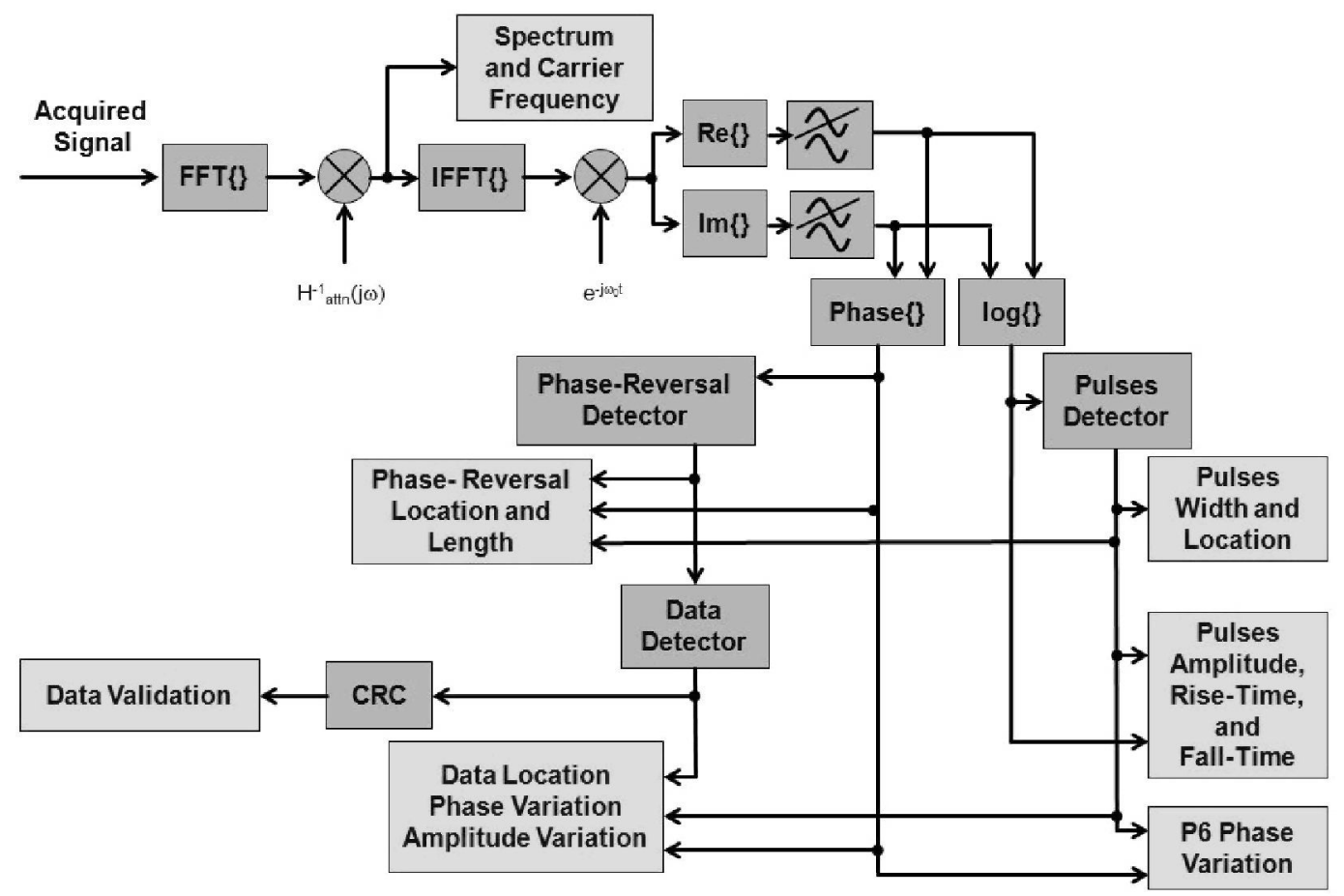

Fig. 3. Signal processing scheme.

TABLE I

Mode S INTERrogation WAVEForm Parameters

\begin{tabular}{|c|c|c|}
\hline PARAMETER & NOMINAL VALUE & TOLERANCE \\
\hline \hline P1 and P2 pulse Width & $800 \mathrm{~ns}$ & $\pm 100 \mathrm{~ns}$ \\
\hline P6 Pulse Width & $16.25 \mu \mathrm{s}$ (Short) & $\pm 250 \mathrm{~ns}$ \\
\hline P1 to P2 Space & $20.25 \mu \mathrm{s}$ (Long) & $\pm 50 \mathrm{~ns}$ \\
\hline Pulse Rise Time & - & $50 \mathrm{~ns}$ to $100 \mathrm{~ns}$ \\
\hline Pulse Fall Time & - & $50 \mathrm{~ns}$ to $200 \mathrm{~ns}$ \\
\hline P2 Rise to Phase Reverse & $2.75 \mu \mathrm{s}$ & $\pm 20 \mathrm{~ns}$ \\
\hline P6 Rise to Phase Reverse & $1.25 \mu \mathrm{s}$ & $\pm 20 \mathrm{~ns}$ \\
\hline Phase Change Duration & - & $<80 \mathrm{~ns}$ \\
\hline Data phase reversal & $(\mathrm{N} \times 250 \mathrm{~ns})(\mathbf{N} \leq 2)$ & $\pm 20 \mathrm{~ns}$ \\
\hline Guard time after last data & - & $>500 \mathrm{~ns}$ \\
\hline $\begin{array}{c}\text { P6 Amplitude } \\
\text { (1st } \mu \text { s ) }\end{array}$ & - & $>(\mathrm{P} 1 \mathrm{Amplitude})-0.25 \mathrm{~dB}$ \\
\hline $\begin{array}{c}\text { P6 Amplitude Variation } \\
\text { (Total) }\end{array}$ & - & $\pm 1 \mathrm{~dB}$ \\
\hline $\begin{array}{c}\text { P6 Amplitude Variation } \\
\text { (between successive chips) }\end{array}$ & - & $\pm 0.25 \mathrm{~dB}$ \\
\hline P6 Maximum phase variation & - & $<370^{\circ}$ \\
\hline Phase variation per chip & $180^{\circ}$ & $\pm 5^{\circ}$ \\
\hline Frequency & $1030 \mathrm{MHz}$ & $\pm 0.01 \mathrm{MHz}$ \\
\hline
\end{tabular}

samples and stores the transmitted signal. The test setup is shown in Fig. 2.

\section{Signal Processing}

In this paper, the oscilloscope only acquires the $60-\mathrm{MHz}$ IF signal and all the signal processing stage is carried out on a personal computer using MATLAB. First, the sampled and stored signal is corrected due to the fact that an attenuator has been placed before the mixer, which was done to protect it from high-power signals generated by the Mode $\mathrm{S}$ interrogator module. After that, the spectrum is obtained through an FFT, and the carrier frequency value and the spectrum mask [4] are checked. Next, the signal is digitally downconverted to baseband, where the real and imaginary parts of the resulting complex signal are extracted

$$
\begin{aligned}
& I=\mathfrak{K}\left(S(t) e^{-j \omega_{0} t}\right) \\
& Q=\Im\left(S(t) e^{-j \omega_{0} t}\right)
\end{aligned}
$$

where

$$
\omega_{0}=2 \pi \cdot 60 \cdot 10^{6} \mathrm{rad} / \mathrm{s} .
$$

The obtained I/Q signals can be low-pass filtered and subsampled without degradation of the information or signal shape. To subsample the signal reduces the required computational burden. The RF bandwidth defined by 


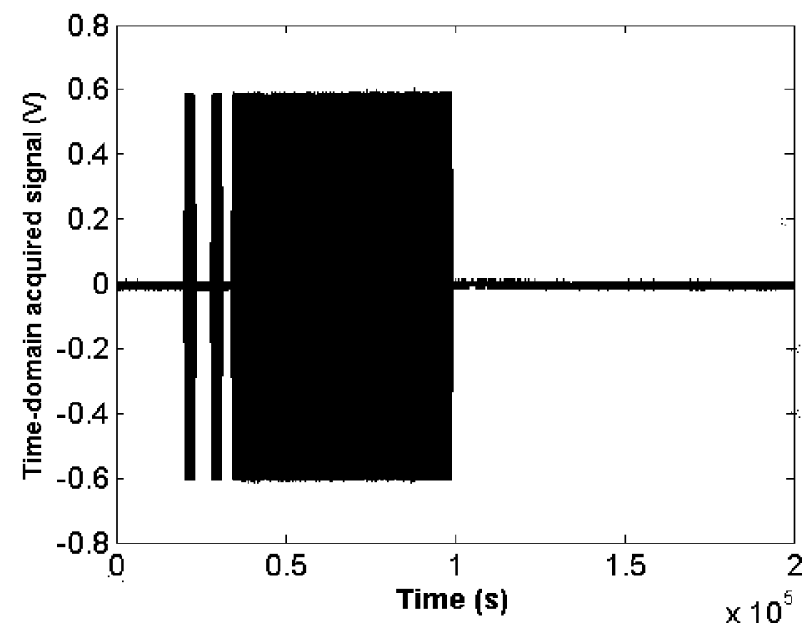

Fig. 4. Time domain of the laboratory generator short Mode $\mathrm{S}$ interrogation signal.

ICAO is $8 \mathrm{MHz}$, so a $10-\mathrm{MHz}$ low-pass filter is used to filter baseband I/Q signals. This filter does not affect the spectrum of the signal of the transmitter and it is able to eliminate the undesired mixers.

Mode $S$ regulation defines the amplitude requirements in decibels and the phase ones in degrees. Thus, a logarithmic amplitude detector is used for the amplitude (video signal), while inverse tangent of I/Q for the phase (phase signal). The video signal is used for pulse measurements (i.e., width, relative position, length, amplitude, rise time, and fall time) and the phase signal is used for the phase-reversal position and length, allowing data extraction and validation.

The signal processing scheme is shown in Fig. 3. By achieving a complete detection and demodulation, all the parameters defined by ICAO (see Table I) can be measured and checked with the required precision and tolerance, and this can be carried out by using general-purpose equipment.

The greatest advantages of this scheme (see Fig. 3) are that the measurement process is carried out fully automatically and that it can perform any specific measurement defined by the user. The system can be configured for measuring, checking parameters, and storing the results as long as the user wants. Furthermore, if a temperature or pressure chamber is available, tasks such as controlling the chamber by a personal computer and performing automatic measurements, while the ambient conditions are controlled, can also be conducted.

\section{EXPERIMENTAL RESUlts}

The test setup presented in this paper has been used to test three different models of Mode $\mathrm{S}$ interrogator transmitters. The first one is a high-quality laboratory generator, and the second ones are actual Mode $\mathrm{S}$ transmitters that have some well-known problems on their signal synthesizers. These models are used to provide valid and nonvalid signals to check and validate the test setup.

\section{A. High-Quality Laboratory Generator}

The acquired data of the first Mode $\mathrm{S}$ transmitter, which complie with all ICAO [4] requirements, are described in the following lines.

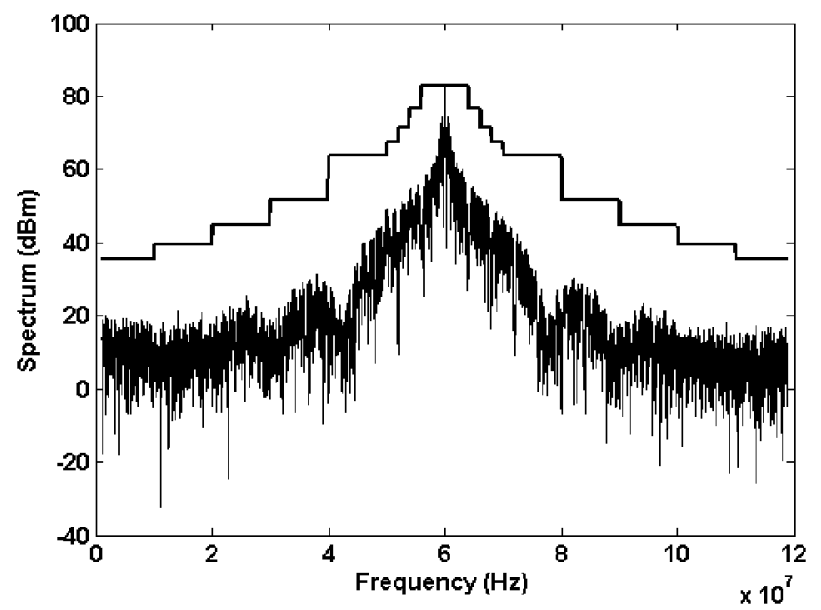

Fig. 5. Spectrum of the laboratory generator short Mode S interrogation signal.

The IF signal is directly acquired using the oscilloscope and its time-domain representation is shown in Fig. 4.

This signal is processed, the signal spectrum is obtained, and it is compared with the spectrum mask defined by ICAO (Fig. 5).

All the parameters of the signal, including the phase evolutions, are obtained using MATLAB and the information data are decoded. With the signal envelope, the pulse position and the rise and fall times are checked. The phase reversal is detected from the phase evolution and the data extraction is synchronized with its position. The envelope of the signal and the data detection samples are shown in Fig. 6.

A more detailed image of the phase of $\mathrm{P} 6$, including the sampling points, is shown in Fig. 7. The first vertical line is the phase-reversal detection, and all the following ones are the data sampling points.

The polar representation of the P6 I/Q evolution is shown in Fig. 8. This representation provides a good visual overview of the signal quality.

The signal from the high-quality laboratory generator complies with all the parameters defined for Mode S interrogation and the obtained values are in accordance with the programmed ones. As a summary of the signal analysis, some parameters are pointed to be compared with the ones of the faulty transmitter:

1) P6 amplitude variation: $0.41 \mathrm{~dB}$;

2) phase reverse duration: $75 \mathrm{~ns}$;

3) maximum phase variation in $\mathrm{P} 6: 365^{\circ}$;

4) data-phase position error: $1 \mathrm{~ns}$;

5) maximum amplitude variation per chip: $0.09 \mathrm{~dB}$;

6) center frequency: $60.000 \mathrm{MHz}$.

\section{B. Actual Mode S Transmitters With Some Problems on Their Signal Synthesizers}

The signal from the first defective Mode $\mathrm{S}$ transmitter was acquired using the proposed test setup and processed using MATLAB. Some of the most relevant information is shown 

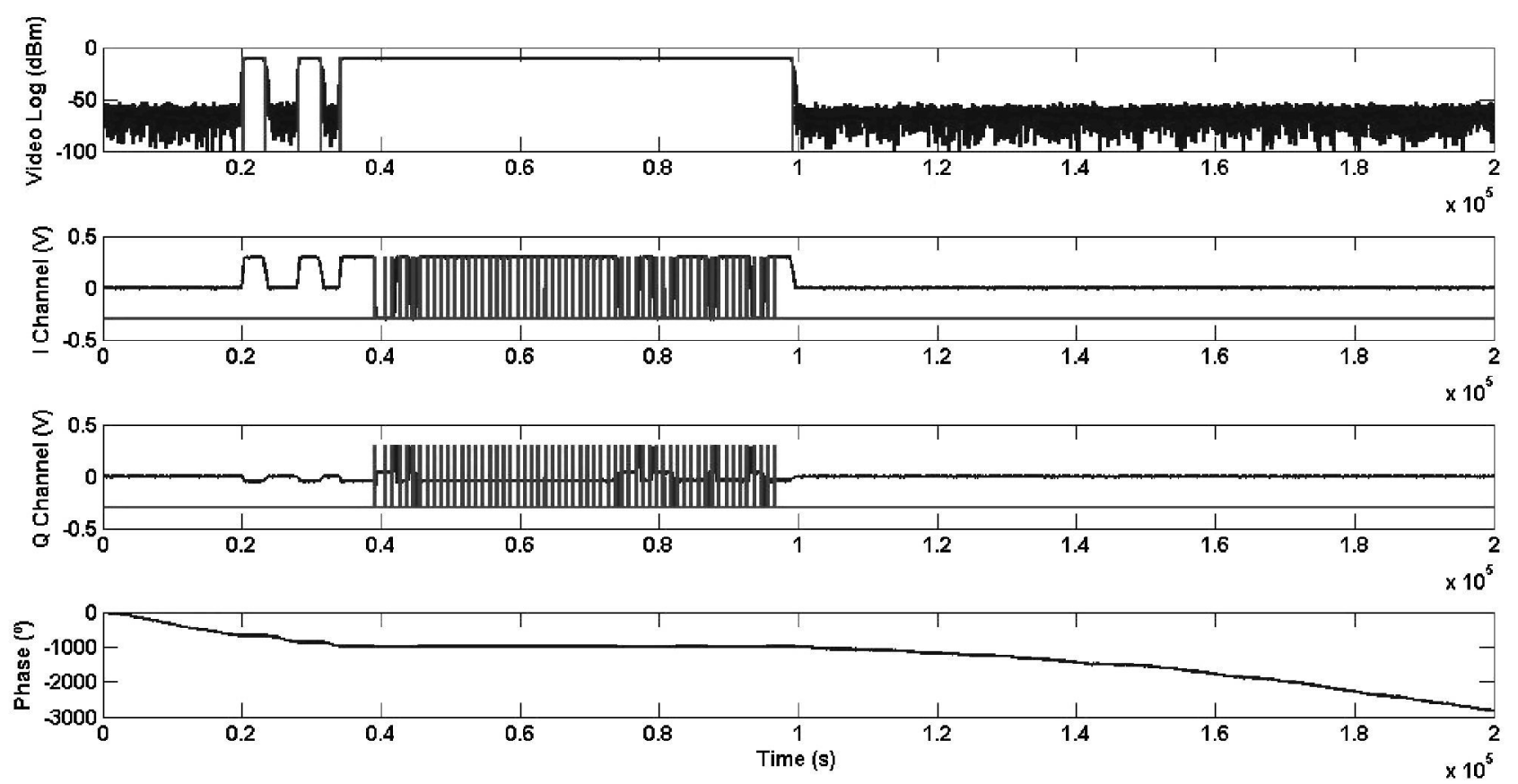

Fig. 6. Laboratory generator signal envelope, I/Q, and phase.

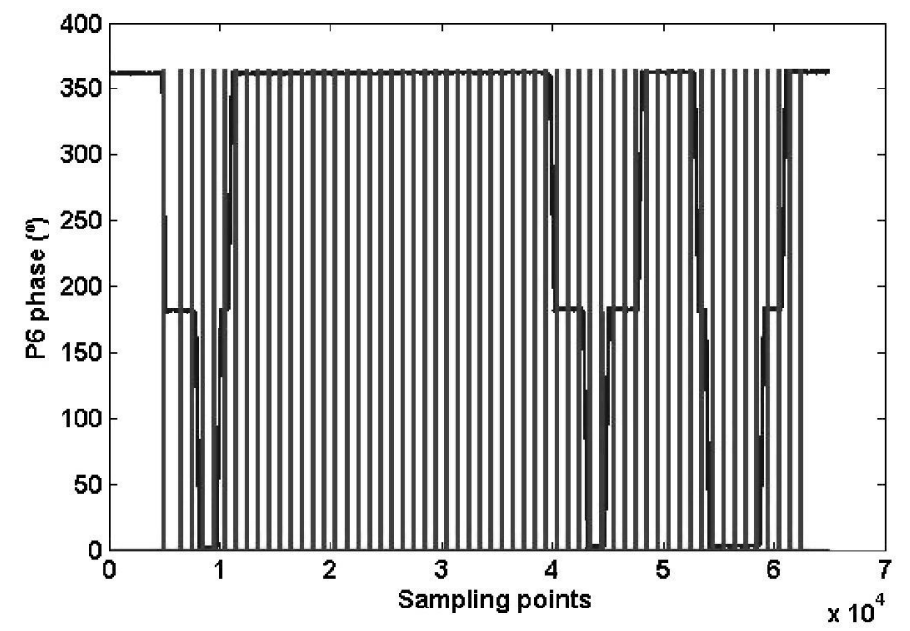

Fig. 7. Laboratory generator P6 phase.

in Figs. 9 and 10. The envelope amplitude of the signal and the phase are shown in Fig. 9. In the detailed phase evolution of the data area of the signal (P6) (Fig. 10), it can be observed that this modulator presents a problem in the phase response.

The phase signal shown in Fig. 10 has phase transition problems such as overshooting and frequency error as well. The polar representation of the P6 I/Q evolution is shown in Fig. 11. It shows the phase error in bits and the envelope variation of the signal.

After the full analysis of the transmitted signal of the Mode $\mathrm{S}$ transmitter under test, the parameters of the ICAO specifications were measured accurately. The parameters that did not meet the specifications are

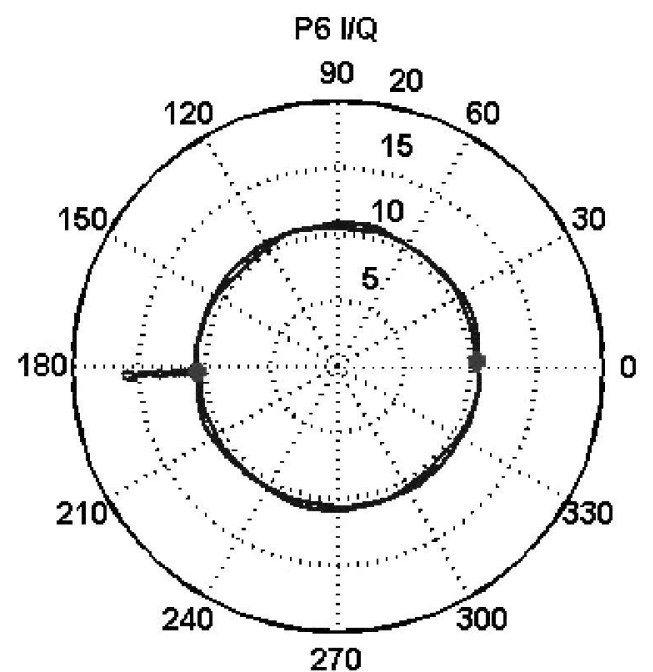

Fig. 8. Laboratory generator P6 I/Q.

marked as fault:

1) P6 amplitude variation: $1.81 \mathrm{~dB}$ (FAULT);

2) phase reverse duration: $118 \mathrm{~ns}$ (FAULT);

3) maximum phase variation in P6: $404^{\circ}$ (FAULT);

4) data-phase position error: $15 \mathrm{~ns}(\mathrm{OK})$;

5) maximum amplitude variation per chip: $0.7 \mathrm{~dB}$ (FAULT);

6) center frequency: $60.006 \mathrm{MHz}(\mathrm{OK})$.

Other defective Mode $\mathrm{S}$ transmitter resulted in the phase shown in Fig. 12. Some problems with phase transitions may be detected with the signal spectrum. But others like the one shown in Fig. 12 can only be detected by conducting a more complex phase analysis. The signal met all the requirements 

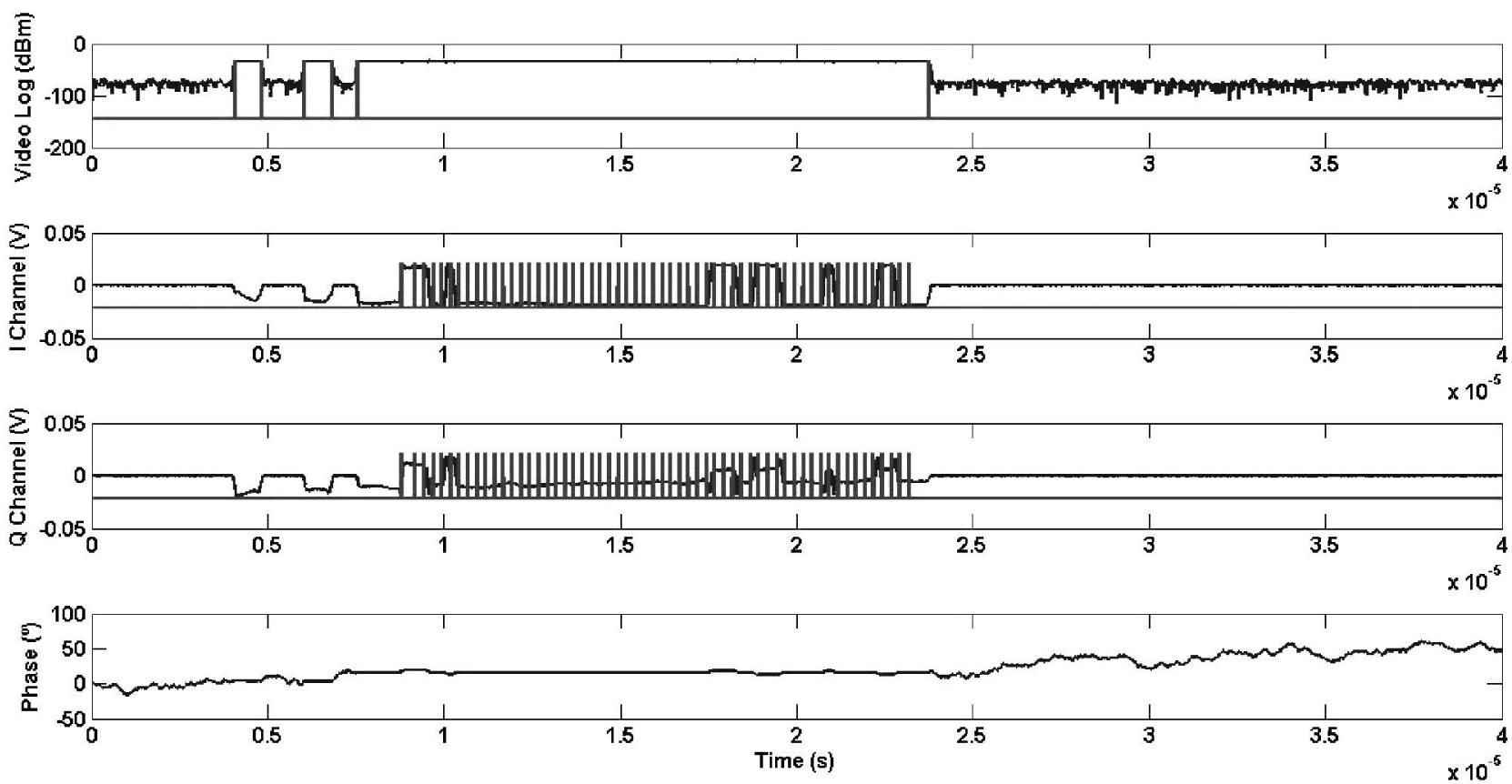

Fig. 9. First Mode $\mathrm{S}$ transmitter signal envelope, I/Q, and phase.

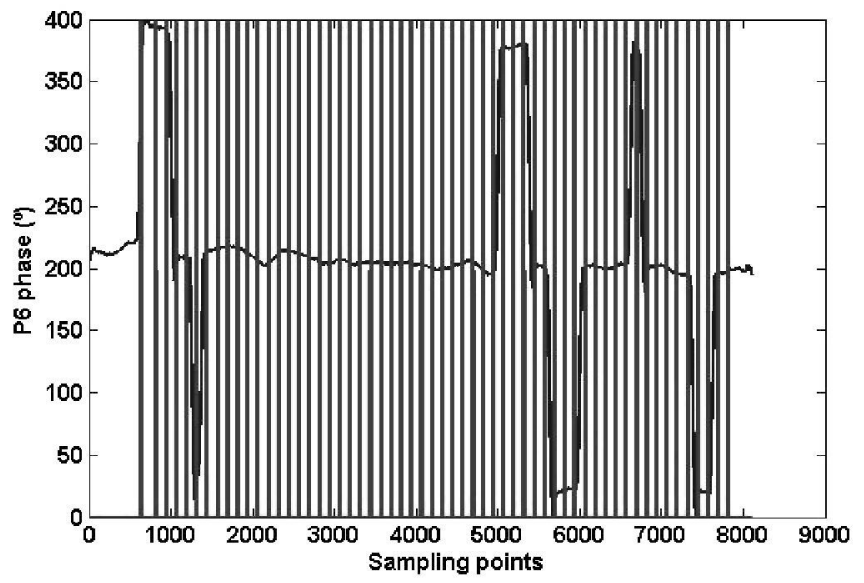

Fig. 10. First Mode S transmitter P6 phase.

but maximum phase variation in P6. In spite of the fact that this signal was generated at nominal frequency, it presented spectrum degradation due to the fact that the phase derivation in the phase changes was always negative. If the phase of the signal is not studied in detail, this problem can be interpreted as a problem with the collector modulation, because most of the demodulators are able to demodulate this defective phase modulation providing correct data. When a demodulator is working with a high $\mathrm{S} / \mathrm{N}$ ratio, as the high-level test equipment does, the signal is correctly demodulated, but it cannot assure that the signal will be correctly demodulated by a receiver in real media S/N conditions.

\section{Cost of the Proposed System}

Some pieces of high-end equipment, which are designed with a similar purpose to the one presented in this paper, exist

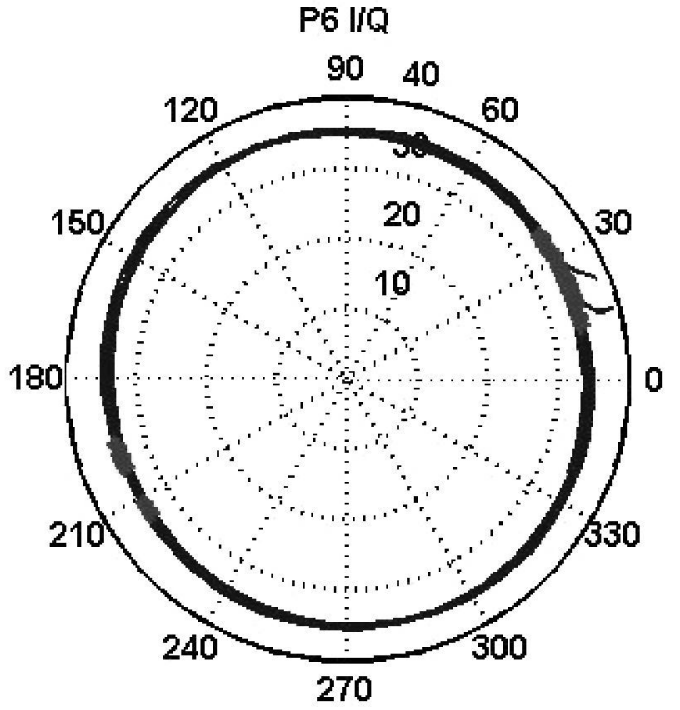

Fig. 11. First Mode S transmitter P6 I/Q.

on the market. Some examples of these high-end equipment are the following: Radar Analysis Support System, with a cost of more than $€ 1000000$; and high-performance signal and spectrum analyzer, with a cost of more than $€ 75000$.

Table II shows that the cost of the system presented in this paper is less than $€ 31000$. This cost compares very favorably to the above-mentioned pieces of high-end equipment that are available on the market. To evaluate the presented cost, it must be taken in consideration that the list of pieces of equipment presented in Table II are general-use equipment. Therefore, the budget is shared with other test purpose, but the pieces of high-end equipment are only for the specific test purpose they are designed. 


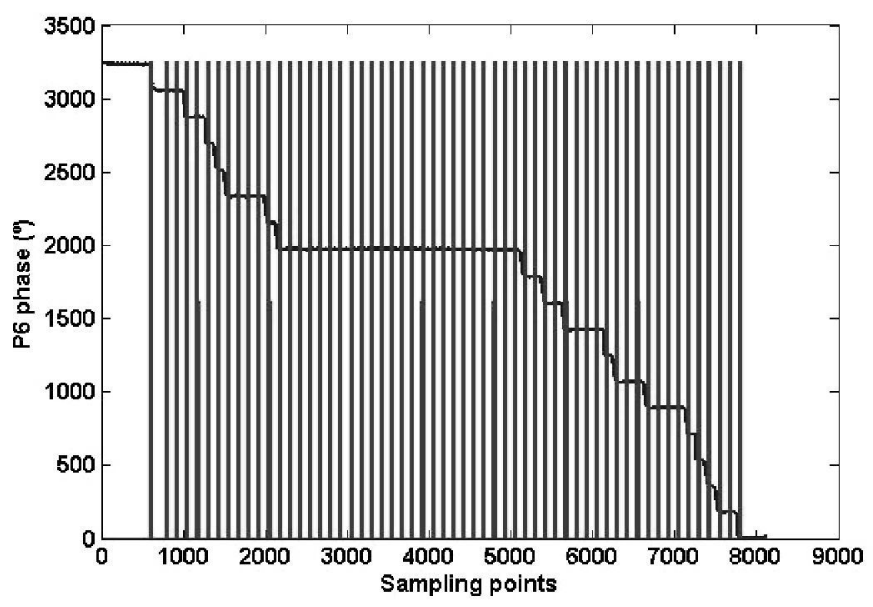

Fig. 12. Second Mode S transmitter phase evolution of P6.

TABLE II

Component Parts of the Proposed S ystem

\begin{tabular}{|c|c|}
\hline Description & Price \\
\hline Coaxial Low Pass Filter BLP-100+ & 28 Euros \\
\hline GPS. Spectracom Epsilon Clock Model EC1S & 2,130 Euros \\
\hline Coaxial Frequency Mixer ZAD-11+ & 53 Euros \\
\hline Digital Oscilloscope TEKTRONIX TDS3034C & 9,610 Euros \\
\hline Signal generator R\&S SMBV100A & 16,443 Euros \\
\hline RF Attenuator NARDA SA12N350A-40 & 1,350 Euros \\
\hline Matlab, Standard License & 2,000 Euros \\
\hline Personal computer & 400 Euros \\
\hline Total Cost: & 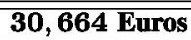 \\
\hline
\end{tabular}

\section{CONClusion}

The proposed test setup provides a low-cost and high-performance system to test and validate Mode S transmitter performances. The proposed technique is able to cover all the ICAO requirements of the modulated signal into a single tool. The setup is able to analyze all Mode S interrogation parameters on an automatic way and perform statistic study of parameters.

Most or even all the pieces of high-level test equipment for MSSR test purpose are focused on operational performances of the radar and they have a demodulator to decode the transmitted signal. They work with an extremely high $\mathrm{S} / \mathrm{N}$ ratio, so they cannot assure proper demodulation in a real operational $\mathrm{S} / \mathrm{N}$ and interference environment. The proposed test setup is oriented to analyze in detail the signal to assure that it has all the robustness, as it is required by ICAO.

The use of a direct IF signal conversion avoids timing calibration and increases the test simplicity, maintenance, and precision. All the processing power of an analysis tool such as MATLAB is available for any detailed analysis or signal modeling that is required. The high acquisition frequency used, $2.5 \mathrm{G} / \mathrm{s}$, provides a 0.4-ns precision on all timing parameter and guarantees this precision for all amplitude to phase measurements without any degradation due to the use of parallel equipments such as a power meter and a differential phase shift keying demodulator.
To illustrate the use of the proposed measurement setup, the results of the performance of two Mode $S$ interrogator transmitters have been shown. The measurement of a reference signal from a laboratory generator with known parameters confirms the accuracy of the proposed setup.

A set of measurements of two Mode $S$ transmitters with some problems was shown and a correct demodulation of the data could be obtained with some demodulator schemas, but the proposed technique allows one to both detect and successfully measure the problems presented by the transmitter under test. Finally, the authors of this paper would also like to say that the proposed method presented in this paper has been successfully used to analyze and verify the signals transmitted by the aviation transponder interrogation Mode 5, distance measuring equipment, and Doppler very high-frequency omnidirectional range equipment.

\section{ACKNOWLEDGMENT}

The authors would like to thank M. Á. del Casar Tenorio for his personal and great contributions to the development of this paper.

\section{REFERENCES}

[1] C. Jacovitti, "Performance analysis of monopulse receivers for secondary surveillance radar," IEEE Trans. Aerosp. Electron. Syst., vol. AES-19, no. 6, pp. 884-897, Nov. 1983.

[2] J. A. Lord, G. G. Cook, A. P. Anderson, and R. S. Orton, "Diagnostics of an SSR array antenna from the measured near-field intensity using phase retrieval," in Proc. 8th Int. Conf. Antennas Propag., vol. 1. Edinburgh, U.K., Mar./Apr. 1993, pp. 315-318.

[3] T. Ikäheimonen, "Measurement of radar spurious emission with high dynamic range and optimized measurement time," IEEE Trans. Instrum. Meas., vol. 60, no. 3, pp. 1010-1016, Mar. 2011.

[4] International Standards and Recommended Practices-Amnex 10 to the Covention on International Civil Aviation-Aeronautical Telecommunications: Surveillance Radar and Collision Avoidance Systems, 4th ed., ICAO, Jul. 2007. [Online]. Available: http://store1.icao.int/index.php/publications/annexes/10-aeronauticaltelecommunications/annex-10-volume-iv-surveillance-radar-andcollision-avoidance-systems-english-printed-11331.html

[5] J. Tol, P. van Genderen, and L. P. Ligthart, "Improvement of the SSR mode S data link using a distributed ground system," in Proc. CIE Int. Conf. Radar, Beijing, China, Oct. 1996, pp. 519-522.

[6] I. Balajti, "Performance measurements of the radar "in situ," in Proc. Microw., Radar Remote Sens. Symp. (MRRS), Kiev, Ukraine, Sep. 2008, pp. 334-339.

[7] R. M. Trim, "Mode S: An introduction and overview," Electron. Commun. Eng. J., vol. 2, no. 2, pp. 53-59, Apr. 1990.

[8] D. Bonefačić, J. Jančula, and N. Majurec, "Model of a monopulse rada1 tracking system for student laboratory," Radioengineering, vol. 16, no. 3, pp. 62-67, Sep. 2007.

[9] L. Sorokosz, W. Zieniutycz, M. Pergol, and M. Mazur, "Mutual coupling between IFF/SSR microstrip antennas with reduced transversal sizeExperimental study," Radioengineering, vol. 20, no. 1, pp. 284-288, Apr. 2011.

[10] V. A. Orlando, "The mode S beacon radar system," Lincoln Lab. J., vol. 2, no. 3, pp. 345-362, 1989.

[11] P. R. Drouilhet, Jr., "The development of the ATC radar beacon system: Past, present, and future," IEEE Trans. Commun., vol. 21, no. 5, pp. 408-421, May 1973.

[12] Federal Aviation Administration - Advisory Circular N. 120-86. Aircraft Surveillance Systems and Applications. [Online]. Available: http://www.faa.gov/documentLibrary/media/Advisory_Circular/AC\% 20120-86.pdf.

[13] C. L. Cheng, F. R. Chang, L. S. Wang, and K. Y. Tu, "Highly-accurate real-time syntonization by use of a single-frequency receiver with GPS carrier phase," in Proc. 18th Eur. Freq. Time Forum (EFTF), Guildford, U.K., Apr. 2004, pp. 159-166. 
[14] R. Kraus, M. Treanor, W. J. Sarjeant, and R. Dollinger, "Programmable instrumentation for high repetition rate experiments providing control in real time and acquisition of a train of pulses," Rev. Sci. Instrum., vol. 59, no. 7, pp. 1226-1231, Jul. 1988.

[15] H. S. Mir and L. Albasha, "A low-cost high-performance digital radar test bed," IEEE Trans. Instrum. Meas., vol. 62, no. 1, pp. 221-229, Jan. 2013.

[16] L. A. Muth, C. M. Wang, and T. Conn, "Robust separation of background and target signals in radar cross section measurements," IEEE Trans. Instrum. Meas., vol. 54, no. 6, pp. 2462-2468, Dec. 2005.

[17] M. Yeary et al., "Multichannel receiver design, instrumentation, and first results at the national weather radar testbed," IEEE Trans. Instrum. Meas., vol. 61, no. 7, pp. 2022-2033, Jul. 2012.

[18] R. E. Updegraff, "Precise time and frequency control using the Navstar GPS and a disciplined rubidium oscillator," in Proc. 2nd Int. Conf. Freq. Control Synth., Leicester, U.K., Apr. 1989, pp. 92-96.

[19] R. M. Hambly and T. A. Clark, "Critical evaluation of the Motorola M12+ GPS timing receiver vs. the master clock at the United States Naval Observatory, Washington, DC," in Proc. 34th Annu. PTTI Mtg., Reston, VA, USA, Dec. 2002, pp. 109-115. 